\title{
Survey of the COCOON Project ${ }^{\star}$
}

\author{
M.H. Scholl ${ }^{\star \star}$, H.-J. Schek
}

ETH Zürich, CS Department

\author{
ETH Zentrum, CH-8092 Zürich, Switzerland
}

e-mail: \{scholl, schek\}@inf.ethz.ch

\begin{abstract}
The COCOON project was intended to extend the concepts and the architecture of relational database management systems (DBMSs) beyond nested relational to object-oriented ones. Based upon the nested relational DBMS kernel DASDBS, we have built a prototype implementation of the COCOON model. Key characteristics of COCOON are: generic, set-oriented query and update operators similar to relational algebra and SQL updates, respectively; object-preserving semantics of query operators, which allows for the definition of updatable views; a separation of the two aspects of programming language "classes": type vs. collection; predicative description of collections, similar to "defined concepts" in KL-One-like knowledge representation languages; automatic classification of objects and views (positioning in the class hierarchy); physical clustering of subobjects via the use of nested relations as the internal storage structures; support for the optimization of both, the physical DB design and query transformation, by corresponding optimizers.
\end{abstract}

\section{Motivation}

Two observations have been the rationale of the COCOON project: 1) the relational model needs to be generalized to meet the requirements of new applications, and 2) next generation DBMSs need efficient storage structures for complex objects and smart query optimizers. The overall approach of COCOON can best be characterized by the term evolution instead of revolution [SS91a], that is, the guideline has been to try to integrate, in a consistent way, concepts from other fields within computer science into the database context. Examples are structuring primitives from AI knowledge representation (we reviewed several techniques developed there and found that the KL-One direction was best suited for our purposes). Similarly, object-oriented concepts from programming languages had to be adopted for inclusion into databases. In contrast to many other OODBMSs, particularly most commercially available ones, our approach was not to extend an OOPL with persistency and transactions. Rather, we have

* This work has been funded by the Swiss National Research Fund (SNF) since 1989

* Current address: University of Ulm, CS Department, P.O.Box 4066, 7900 Ulm, Germany. e-mail: scholl@informatik.uni-ulm.de 
emphasized the preservation of established DBMS advantages, such as data in dependence, set-oriented, descriptive languages, optimizability, sometimes at the expense of expressiveness. As a result, COOL, the query and update language of COCOON, is not a (computationally) complete language.

\section{The object model}

The COCOON data model with its language COOL is an extension of the nested $\left(\mathrm{NF}^{2}-\right)$ relational algebra developed in the DASDBS project [SS86, SPSW90]. In addition to allowing relational operators to work on hierarchical structures, as is the case in the nested relational algebra, the structures had to be extended in two ways: non-hierarchical structures (networks of shared subobjects) had to be included, as well as generalizations ("ISA-hierarchies").

Object sharing leads to the introduction of object identities, which can be handled in different ways. For example, one can add a new domain of OID values to the data model, tuples with an ID attribute defined over this domain represent objects. While this is the way how objects have to be represented internally, we pursued a more abstract view for the data model level: a distinction between (abstract) objects and concrete data values was introduced (see also [Bee89, Bee90], for example).

Generalization hierarchies are known from the AI (or semantic data modeling) field, they also appear as inheritance hierarchies in object-oriented programming languages (OOPLs). In AI knowledge representation, such hierarchies are described by predicates that allow for the automatic classification (positioning of a new class), the test for subsumption (is class $C_{1}$ a subclass of class $C_{2}$ ?), and the test for realization (is object $o$ a member of class $C$ ?). The focus of AI classification hierarchies is on an intensional (e.g., predicative) description of the extension (the set of all possible members, i.e, the domain in DB terms) of a class. In OOPLs, class hierarchies are a means to achieve reusability (of class definitions and methods). Substitutability of subclass objects for arguments that are restricted to the superclass is an additional benefit. The main focus of class hierarchies in OOPLs is on the inheritance of structure and behavior (attributes and methods) from superclasses to subclasses. Typically, no collection of instances is kept. If so, there is exactly one per class.

The intended use of generalization hierarchies in OODBs is not exactly the same as in these two fields. First of all, databases should clearly distinguish intensional and extensional (or schema and instance) aspects of "classes": the intensional aspect (akin to the schema of a relation) describes the structure, the behavior, and the set of all possible instances (the domain). The extensional aspect (akin to the current set of tuples in a relation) captures a dynamically changing collection of instances. In general, there might be an arbitrary number (zero, one, or more) of collections over the same "schema". Similar to [Bee89, Bee90, HFW90] and others, we introduced the term "type" to denote the intensional aspects, that is, the description of the interface of the objects that are "instance of" that type. The term "class" is used for a collection (an ex- tension) of objects, the "members of" that class. All members of a class have to be instance of the "member type" of the class. Notice that types and classes are defined separately from each other, similar to types and variables in programming languages. In that respect, a COCOON type corresponds to a PL type and a COCOON class to a persistent set variable over this component type in the PL. COCOON classes, however, are more powerful, since they are arranged in a "class hierarchy" that represents a subset constraint. Furthermore, the subset constraint may be specified by a class predicate: a necessary predicate $(N P)$ restricts the possible members of the subclass to at most those members of the superclass(es) that satisfy $N P$. If the subset constraint is a necessary and sufficient predicate $(N S P)$, the subclass represents exactly those members of the superclass(es) that satisfy $N S P$. In the first case, the subclass is called a "someclass", in the second case an "all-class". This terminology is documented by the COOL syntax for class definitions:

[define] class $\langle$ name $\rangle[:\langle$ membertype $\rangle]$ [ \{some|all $\}\langle$ superclass $\rangle[, \ldots]$ [where $\langle$ predicate $\rangle]]$

The types in COCOON are defined by the set of functions that can be applied to the instance objects, that is, COCOON is an object-function model (cf. [WLH90]). In such object models, there is no distinction between stored attributes and computed (retrieval) methods. We have chosen this approach for its simplicity and because it is an elegant way to achieve data independence: whether the values of the functions defined on an object are stored in the internal record representing the object or whether it is derived by some algorithm is transparent to the object model, it is merely a matter of physical database design. Types are arranged in a type lattice, where a subtype inherits all the functions from its supertype(s), that is, the lattice is the subset lattice of the sets of functions associated with the types. It is important to notice that we have a type lattice in the mathematical sense, whereas we have only a (multiple) hierarchy between classes.

To summarize, we list the modeling concepts found in COCOON, details can be found in [SS90a, SLR+92]:

Object: an application-specific abstraction, instance of an abstract object type

Data Value: a concrete, printable or constructed value (integer, string, set, ...)

Type: a set of functions, forming the interface to its instance objects

Class: a collection object, represents a set of objects of the member type, possibly constrained (some-class) or defined (all-class) by a predicate

Subtype: a type that allows to apply more functions to its instances than to instances of the supertype(s); this relationship forms a lattice

Subclass: a class that has a subset of the members of the superclass(es); this relationship forms a multiple hierarchy

View: a derived class, defined by a query

Variable: a variable is declared with a type, it can be used to hold single objects (i.e., as a temporary name), or sets of objects (if declared over a set type), for later reference within the same program. 


\section{The query language COOL}

The starting point for the development of our object algebra was the observation that relational algebra can easily be extended to more complex data models. We had done so for the nested ( $\mathrm{NF}^{2}$-) relational model: when allowing relations to appear repeatedly, all we have to do is to allow the application of algebraic expressions in a similarly nested way. Whenever we have relation-valued attributes in a relation, we can apply relational expressions to them within selections and projections [SS86]. The programming language view onto the relational model is that a family of basic types (such as integers, strings, etc.) can be used to construct composite types using only one type constructor, namely relation (set of record). If we extend this type system to allow orthogonal (i.e., recursive) use of the type constructor, we have nested relations. The obvious extension of the relational operations is to apply them orthogonally. This is exactly what has been done for nested relations in the algebra of [SS86].

COCOON added more concepts to the "type system" (data model): functions instead of attributes, which may be single- or set-valued, collections (classes) that are organized in a hierarchy based on associated predicates, types that are structured in a lattice, and a distinction of data values and abstract objects. All these new concepts had to be incorporated into the query language. The principal approach is rather straightforward: classes as the collection objects are the arguments of queries, functions play the role of attributes. Therefore algebra operators can be applied to classes, since they represent sets of objects. So, a setoriented query language seems no problem. However, two basic problems had to be solved before an object algebra could be defined consistently with the object model:

- What is the result of a query? (object preservation)

As the input to a query is a collection of objects, the closure property of the model requires the output also to be a collection of objects (otherwise algebra operators could not be composed). On the other hand, if objects are system-internal abstractions, we can not show them to users, all we can show are data values related to the objects. Therefore, many other object query languages always return data values.

We have coined the terms "object-preserving" vs. "value-generating" operator semantics for the two choices [SS90a]. An intermediate possibility is to create new result objects ("object-generating semantics). Value-generating semantics are not appropriate for models where "everything is an object"; object-generating semantics do not allow query results to be used as the arguments of update operations, since the result objects, but not the original objectbase objects would be modified. As COCOON was intended to support updatable views that are defined by query expressions, our choice had to be object-preserving query semantics, where result objects are identical to original objectbase objects.

- Where does a query result (e.g., a view) belong in the type lattice and in the class hierarchy? (dynamic classification)
In order to formally define the query language operators, one has to specify what the results are, both, in terms of result type and result collection. When a query defines a view, the latter means to classify the view class in the class hierarchy.

A type system was defined that allows to statically type-check COOL statements and to derive unique result types for all query expressions by corresponding type inference rules. In order to be able to infer result types for union and intersection operations when the arguments have different types, we need a lattice of types to assign the lowest upper bound and greatest lower bound to the results, respectively [SLT91, LS92b, SLR ${ }^{+92}$ ].

Classification of views can not be solved completely, since subsumption of predicates is undecidable in general. Therefore, we had to implement an incomplete classification algorithm that fails to achieve the lowest possible positioning in some cases. It is, of course, guaranteed that no incorrect classifications are derived [SLT91, Ngu91].

The query operators of COOL are:

Selection: a subset of the input objects is derived by a predicate, the result type is the same as the input type

Projection: (object-preserving) the result is the same set of objects, but a supertype of the input type is associated with it, thus only the functions listed in the projections are applicable to the result

Extend: new derived functions can be defined; the result contains the same objects as the input, but associated with a new (sub-) type that includes the derived functions

Union: takes the union of the input sets, the result type is the least upper bound of the input types

Difference: takes the set difference, the result type is that of the first argument

Intersection: intersects the input sets, the result type is the greatest lower bound of the input types

Pick: Chooses one object from a set, the result type is the element type of the input

Extract: (value-generating projection) generates a set of tuples from the input objects, each tuple component is defined by one expression in the extraction list; this is the only operation that is not object-preserving!

In addition to query operators derived from relational algebra, COOL offers generic update operators that can be used to accomplish simple object manipulation tasks, or to construct more complex update methods. There are basic update operators for object creation and destruction, a global assignment $(:=$, for variables and complete functions) and a local assignment (set, for assigning new result values for given arguments of a function), two operations that add and remove existing objects to/from sets and classes. In addition to these more or less standard updates, we have two operators that perform "object evolution", that is, objects can dynamically gain and lose types! In contrast to OOPLs, 
where the lifetime of objects is short (at most one program execution), objectbases store objects over a long period of time (several years). Consequently, the changes in the roles that the modeled real world objects play need to be reflected in the objectbase as well. However, the objects should preserve their identities.

Therefore, we can not fix the relationship between an object and its type as of the time of object creation.

All of the basic updates are single-object operators. They can be applied to a whole set of objects (e.g., a set obtained by a query) by using the set-iterator "apply_to_all" [LS92a]. As is known for long in the relational context, it is nontrivial to combine set-orientation and updates, since non-deterministic effects arise very easily. We have developed a formal model for "set-oriented" updates which essentially restricts updates to non-conflicting ones in order to be applied in a set-oriented fashion. The notion of conflict that is used here corresponds to conflicts in semantic or multi-level concurrency control [Wei86, Wei88, WH91].

\section{Meta modeling and schema evolution}

The COCOON model is powerful enough to represent COCOON schemata, that is, as a part of each COCOON objectbase, we have a meta schema that comprises information about the types, classes, and functions that have been defined in the schema. These meta objects not only serve as the "data dictionary" or objectbase catalog, they are also used as the basis for schema evolution. The COOL update operators can also be applied to the meta objects, so as to express schema modifications [TS92]. The problem attacked here was to define the update operations and the meta types/classes in such a way that schema modification automatically propagate to the instance level. Of course, an implementation will avoid eager transformation and try to use views, schema versions, or lazy transformation of the instance objects to avoid load peaks at schema modification time.

\section{System architecture and optimization}

The target architecture of the COCOON implementation uses the nested relational DBMS kernel DASDBS [SPSW90] as the storage manager. This allows for hierarchical clustering techniques that can be exploited to reduce the amount of physical disk I/O when large structured objects are loaded into main memory. An overview of this mapping approach is contained in the article "COCOON and KRISYS - a comparison" in this volume, a more detailed discussion is contained in [DHL $\left.{ }^{+} 92, \mathrm{Sch} 92 \mathrm{~b}\right]$. A prototype of a physical database design tool has been implemented to aid the database administrator in selecting a good physical design for a given COOL objectbase and load description.

At query processing time, the query optimizer has to transform COOL queries into execution plans that consist of DASDBS kernel calls and higher-level query processing strategies, such as joins and address dereferencing [RS92]. This transformation is also exemplified in the article "COCOON and KRISYS - a com- parison" in this volume. The EXODUS query optimizer generator [GD87] was used to build parts of the COOL query optimizer.

In addition to the DASDBS realization of COCOON, we have built two further prototypes in order to allow comparisons, both qualitative (how difficult is the implementation?) and quantitative (performance experiments): one uses the ONTOS object-oriented DBMS product, the other one the Oracle RDBMS [TS91]. Particularly the Oracle vs. DASDBS experiments are intended to evaluate the effects of hierarchical clustering, since Oracle allows for the definition of "clusters" that can simulate two-level nested relations. To date, we can not report on the performance experiments yet, some qualitative experiences with the two commercial platforms have been given in [TS91], results concerning the DASDBS realization are given in [TRSB92, Lue92].

\section{Recursive query processing algorithms}

In the context of a nested relational storage manager, we have also carried out investigations on the use and the adaptation of graph algorithms for recursive query processing in the database context. In contrast to classical analyses of graph algorithms (transitive closure, shortest paths, etc.), we had to take physical $\mathrm{I} / \mathrm{O}$ costs into account, since the data representing the graph is in the objectbase. New algorithms have been designed, implemented, and extensively tested and evaluated in comparison with various others [Jia90a, Jia92c].

\section{Publications}

Outside publications: [HS91, Jia89, Jia90b, Jia91, Jia92a, Jia92b, LS92b, SLT91, SLT90, SS90a, SS90b, SS91b, SS91a, SST92, SSW91, Sch92a, Sch92b, Tre91, TS92]

Internal reports and non-refereed publications: [DHL ${ }^{+92, ~ J i a 90 a, ~ L S 92 c, ~ R R S 92, ~}$ RS92, TRSB92, TS91]

Theses: [Boo91, Fie91, Fra91, Fri91, Gar91, Ger90, Göh91, Gro91, Jia92c, Kla91, Kla90, Kri90, Lae91, Loe91, Lue92, Mes91, MW89, Ngu91, Rec90, Vet89, Vet90, Wic91, Wil91]

\section{Acknowledgements}

The COCOON project belongs to the research area "Architecture of ObjectBases" investigated jointly with the group around Theo Härder (see the articles "COCOON and KRISYS - a comparison" and "KRISYS - a KBMS supporting the development and processing of advanced applications" in this volume). In the COCOON project, Christian Laasch, Christian Rich, and Markus Tresch have worked on the formalization, optimization, and meta modeling, respectively. Bin 
Jiang investigated graph algorithms for recursive query processing. Numerous students have pushed forward the prototype implementation, they are listed with their theses above. Currently, work on COCOON is continued at the University of Ulm in cooperation with ETH Zürich, since M. Scholl, C. Laasch, C. Rich, and M. Tresch are now in Ulm.

\section{References}

[Bee89] C. Beeri. Formal models for object-oriented databases. In W. Kim, J.M. Nicolas, and S. Nishio, editors, Proc. 1st Int'l Conf. on Deductive and Object-Oriented Databases, pages 370-395, Kyoto, December 1989. NorthHolland. Revised version appeared in "Data \& Knowledge Engineering", Vol. 5, North-Holland.

[Bee90] C. Beeri. A formal approach to object-oriented databases. Data \& Knowledge Engineering, 5:353-382, October 1990.

[Boo91] M. Boos. Realisierung eines COOL-Parsers. Diplomarbeit, Departement Informatik, ETH Zürich, March 1991.

[DHL ${ }^{+}$92] S. Dessloch, T. Härder, F.-J. Leick, N.M. Mattos, C. Laasch, C. Rich, M.H. Scholl, and H.-J. Schek. KRISYS and COCOON: A survey and comparison. Technical report, ZRI Report, University of Kaiserslautern, 1992.

[Fie91] B. Fiechter. Prococoon - Erweiterung einer grafischen Schnittstelle zu COCOON. Diplomarbeit, Departement Informatik, ETH Zürich, September 1991.

[Fra91] J. Frattaroli. Entwurf und Implementierung eines Übersetzers für Wegeanfragen. Diplomarbeit, Departement Informatik, ETH Zürich, March 1991.

[Fri91] M. Fritsch. Implementierung der Abbildung von COCOON auf das ooDBMS ONTOS. Diplomarbeit, Departement Informatik, ETH Zürich, March 1991.

[Gar91] S. Garazi. Mapping cool queries and updates to oracle-sql. Diplomarbeit, Departement Informatik, ETH Zürich, September 1991.

[GD87] G. Graefe and D. J. DeWitt. The EXODUS optimizer generator. In Proc. ACM SIGMOD Conf. on Management of Data, pages 160-172, San Francisco, May 1987. ACM.

[Ger90] A. Gericke. Precompiler für NF2 Anfragen in C-Programmen. Diplomarbeit, Departement Informatik, ETH Zürich, August 1990.

[Göh91] A. Göhring. Adaptiver physischer DBEntwurf für objektorientierte Datenbanksysteme. Diplomarbeit, Departement Informatik, ETH Zürich, September 1991 .

[Gro91] R. Gross. Physischer DB-Entwurf für objektorientierte Datenbanken. Diplomarbeit, Departement Informatik, ETH Zürich, March 1991.

[HFW90] A. Heuer, J. Fuchs, and U. Wiebking. OSCAR: An object-oriented database system with a nested relational kernel. In Proc. Int'l Conf. on Entity-Relationship Approach, Lausanne, Switzerland, October 1990. NorthHolland. to appear.

[HS91] A. Hewer and M.H. Scholl. Principles of object-oriented query languages. In H.-J. Appelrath, editor, Proc. GI Conf. on Database Systems for Office, Engineering, and Scientific Applications, pages 178-197, Kaiserslautern, March 1991. IFB 270, Springer Verlag, Heidelberg.
[Jia89] B. Jiang. Making the partial transitive closure an elementary database operation. In Proceedings of the GI/SI Conference on Database Systems for Office Automation, Engineering, and Scientific Applications, pages 231-245, Zurich, March 1989. Spring-Verlag, IFB 204.

[Jia90a] B. Jiang. Design, analysis, and evaluation of algorithms for computing partial transitive closures in databases. Technical Report 132, ETH Zurich, Zurich, Schwitzerland, June 1990.

[Jia90b] B. Jiang. A suitable algorithm for computing partial transitive closures in databases. In Proceedings of the IEEE International Conference on Data Engineering, pages 264-271, 1990.

[Jia91] B. Jiang. Traversing graphs in a paging environment, BFS or DFS? Information Processing Letters, 37(3):143-147, 1991.

[Jia92a] B. Jiang. DFS-traversing graphs in a paging environment, LRU or MRU? Information Processing Letters, 40(4):193-196, 1992.

[Jia92b] B. Jiang. I/O-efficiency of shortest path algorithms: A analysis. In Proceedings of the IEEE International Conference on Data Engineering, pages $12-19,1992$.

[Jia92c] B. Jiang. Pfad-Algorithmen für Datenbank Systeme. Doktorarbeit, Departement Informatik, ETH Zürich, 1992.

[Kla90] H. Klas. Analysis of parallel algorithms for computing partial transitive closures by simulation. Semesterarbeit, Departement Informatik, ETH Zürich, August 1990.

[Kla91] H. Klas. Implementierung und Evaluierung von Graphenalgorithmen für Wegeprobleme auf ORACLE. Diplomarbeit, Departement Informatik, ETH Zürich, August 1991.

[Kri90] R. Kritzer. Evaluierung verschiendener Joinalgorithmen auf einem DBSKern nach dem NF2-Relationenmodell. Diplomarbeit, Departement Informatik, ETH Zürich, March 1990.

[Lae91] T. Laes. Generierung und Evaluierung eines Anfrageoptimierers. Diplomarbeit, Departement Informatik, ETH Zürich, August 1991.

[Loe91] M. Loeliger. Co2on - Übersetzung von Anfragen der Sprache COOL auf das objektorientierte Datenbanksystem ONTOS. Diplomarbeit, Departement Informatik, ETH Zürich, September 1991.

[LS92a] C. Laasch and M.H. Scholl. Deterministic semantics of set-oriented update sequences. Submitted for Publication, 1992.

[LS92b] C. Laasch and M.H. Scholl. Generic update operations keeping objectoriented databases consistent. In Proc. 2nd GI Workshop on Information Systems and Artificial Intelligence (IS/KI), Ulm, February 1992. Springer IFB, Heidelberg.

[LS92c] C. Laasch and M.H. Scholl. Set-oriented update sequences for an OO-Model. In U.W. Lipeck and R. Manthey, editors, Proc. 4th GI Workshop on Foundations of Database Systems, Barsinghausen, Germany, June 1992. ECRC Munich, Germany, Technical Report ECRC-92-13.

[Lue92] J.M. Luthi. Evaluierung verschiedener Joinalgorithmen auf einem NF2 DBS-Kern. Semesterarbeit, Departement Informatik, ETH Zürich, 1992.

[Mes91] F. Meschberger. Implementierung der Abbildung von COCOON auf ORACLE. Diplomarbeit, Departement Informatik, ETH Zürich, March 1991.

[MW89] B. Monnerat and K. Warszas. Darstellung und graphische Anfrageformulierung für semantische Netze. Diplomarbeit, Departement Informatik, ETH Zürich, February 1989 
[Ngu91] H.-M. Nguyen. Einordnung von Klassen und Views in eine KlassenHierarchie. Diplomarbeit, Departement Informatik, ETH Zürich, July 1991.

[Rec90] U. Recker. Implementierung eines Algorithmus zur Berechnung der Partiellen Transitiven Hülle auf dem DASDBS-Datenbankkernsystem. Diplomarbeit, Departement Informatik, ETH Zürich, August 1990.

[RRS92] A. Rosenthal, C. Rich, and M.H. Scholl. Reducing duplicate work in relational join(s): A unified approach. Technical Report 172, ETH Zurich, Dept. of Computer Science, January 1992.

[RS92] C. Rich and M.H. Scholl. Query optimization in COCOON. In U.W. Lipeck and R. Manthey, editors, Proc. 4th GI Workshop on Foundations of Database Systems, Barsinghausen, Germany, June 1992. ECRC Munich, Germany, Technical Report ECRC-92-13.

[Sch92a] M.H. Scholl. Extensions to the relational data model. In P. Loucopoulos and R. Zicari, editors, Conceptual Modelling, Databases and CASE: An Integrated View of Information Systems Development. Jon Wiley \& Sons, New York, 1992. To appear.

[Sch92b] M.H. Scholl. Physical database design for an object-oriented database system. In J.C. Freytag, G. Vossen, and D.E. Maier, editors, Query Processing for Advanced Database Applications. Morgan Kaufmann, Los Altos, Ca., 1992. To appear.

[SLR + 92] M.H. Scholl, C. Laasch, C. Rich, H.-J. Schek, and M. Tresch. The COCOON object model. Technical report, ETH Zürich, Dept. of Computer Science, 1992. In preparation.

[SLT90] M.H. Scholl, C. Laasch, and M. Tresch. Views in object-oriented databases. In Proc. 2nd Int'l GI Workshop on Foundations of Models and Languages for Data and Objects, Aigen, Austria, September 1990. Techn. Report 90/3, TU Clausthal, Germany.

[SLT91] M.H. Scholl, C. Laasch, and M. Tresch. Updatable views in object-oriented databases. In C. Delobel, M. Kifer, and Y. Masunaga, editors, Proc. 2nd Int'l Conf. on Deductive and Object-Oriented Databases (DOOD), pages 189-207, Munich, December 1991. LNCS 566, Springer, Heidelberg.

[SPSW90] H.-J. Schek, H.-B. Paul, M.H. Scholl, and G. Weikum. The DASDBS project: Objectives, experiences and future prospects. IEEE Trans, on Knowledge and Data Engineering, 2(1):25-43, March 1990. Special Issue on Database Prototype Systems.

[SS86] H.-J. Schek and M. H. Scholl. The relational model with relation-valued attributes. Information Systems, 11(2):137-147, jun 1986.

[SS90a] M.H. Scholl and H.-J. Schek. A relational object model. In S. Abiteboul and P.C. Kanellakis, editors, ICDT '90 - Proc. Int'l. Conf. on Database Theory, pages 89-105, Paris, December 1990. LNCS 470, Springer Verlag, Heidelberg.

[SS90b] M.H. Scholl and H.-J. Schek. A synthesis of complex objects and objectorientation. In Proc. IFIP TC2 Conf. on Object Oriented Databases (DS-4), Windermere, UK, July 1990. North-Holland. To appear.

[SS91a] H.-J. Schek and M.H. Scholl. From relations and nested relations to object models. In M.S. Jackson and A.E. Robinson, editors, Aspects of Databases - Proc. 9th British Nat. Conf. on Databases, pages 202-225, Wolverhampton, UK, July 1991. Butterworth-Heinemann, Oxford.

[SS91b] M.H. Scholl and H.-J. Schek. Supporting views in object-oriented databases. IEEE Database Engineering Bulletin, 14(2):43-47, June 1991. Special Issue on Foundations of Object-Oriented Database Systems.

[SST92] M.H. Scholl, H.-J. Schek, and M. Tresch. Object algebra and views for multiobjectbases. In Proc. Int'l Workshop on Distributed Object Management, Edmonton, Canada, August 1992. Morgan Kaufmann.

[SSW91] H.-J. Schek, M.H. Scholl, and G. Weikum. The background of the DASDBS \& COSMOS projects. In Proc. Int'l Conf. on Mathematical Foundations of Database Systems (MFDBS), Rostock, Germany, May 1991. LNCS, Springer Verlag, Heidelberg.

[Tre91] M. Tresch. A framework for schema evolution by meta object manipulation. In Proc. 3rd Int'l Workshop on Foundations of Models and Languages for Data and Objects, Aigen, Austria, September 1991.

[TRSB92] W.B. Teeuw, C. Rich, M.H. Scholl, and H.M. Blanken. An Evaluation of Physical Disk I/Os for Complex Object Processing. Technical report, ETH Zürich, Dept. of Computer Science, 1992.

[TS91] M. Tresch and M.H. Scholl. Implementing an object model on top of commercial database systems. In M. Scholl, editor, Proc. 3rd GI Workshop on Foundations of Database Systems, Volkse, Germany, May 1991. Techn. Report 158, Dept. of Computer Science, ETH Zürich.

[TS92] M. Tresch and M.H. Scholl. Meta object management and its application to database evolution. In Proc. 11th Int'l Conf. Entity-Relationship Approach, Karlsruhe, Germany, October 1992. Springer.

[Vet89] M. Vetsch. Graphische Charakteristika von Relationen. Semesterarbeit, Departement Informatik, ETH Zürich, August 1989.

[Vet90] M. Vetsch. Graphische Charakteristika binärer Relationen in existierenden Datenbanken. Diplomarbeit, Departement Informatik, ETH Zürich, February 1990.

[Wei86] G. Weikum. Transaktionsverwaltung in Datenbanksystemen mit Schichtenarchitektur. Dissertation, Fachbereich Informatik, Technische Hochschule Darmstadt, Dezember 1986.

[Wei88] W.E. Weihl. Commutativity-based concurrency control for abstract data types. IEEE Transactions on Computers, 37(12), 1988.

[WH91] G. Weikum and C. Hasse. Multi-level transaction management for complex objects: Implementation, performance, parallelism. Technical Report 162, ETH Zürich, Dept. of Computer Science, July 1991.

[Wic91] P. Wickli. Graphische Schnittstelle zu COCOON. Diplomarbeit, Departement Informatik, ETH Zürich, March 1991.

[Wil91] D. Wilhelm. Query-Schnittstelle fuer DASDBS. Diplomarbeit, Departement Informatik, ETH Zürich, September 1991.

[WLH90] K. Wilkinson, P. Lyngbaek, and W. Hasan. The Iris architecture and implementation. IEEE Trans. on Knowledge and Data Engineering, 2(1):63-75, March 1990. Special Issue on Prototype Systems. 